\title{
Neutrophil elastase inhibitor prevents ischemic brain damage via reduction of vasogenic edema
}

\author{
Yuka Ikegame $^{1,3}$, Kentaro Yamashita ${ }^{1,3}$, Shin-ichiro Hayashi ${ }^{2}$, Shin-ichi Yoshimura ${ }^{1}$, Shigeru Nakashima ${ }^{2}$ \\ and Toru Iwama ${ }^{1}$
}

Release of neutrophil elastase is one of the harmful inflammatory reactions in acute cerebral ischemia. Therefore, inhibition of elastase released from neutrophils could be a useful strategy for the treatment of acute stroke. To evaluate this hypothesis, the effect of sivelestat, a selective neutrophil elastase inhibitor was examined in a mouse model of focal ischemia. The results obtained indicate that sivelestat reduced brain edema and vascular permeability, and subsequently improved the neurological deficit in an acute focal ischemia. The architecture of microvessels was analyzed by identifying vascular endothelial cells, which were prelabeled by injecting fluorescein-labeled Griffonia simplicifolia lectin I-isolectin B4 into a tail vein. Most of the microvessels in the infarcted area were structurally destroyed in the control group. In sharp contrast, microvessels in the boundary zone were well maintained in the sivelestat-treated group. Moreover, the expression of angiopoietin-1 was elevated at the ischemic margin in the sivelestat-treated group. Furthermore, the neutrophil elastase inhibitor rescued human brain microvascular endothelial cells in culture from neutrophil elastase-induced damage. These results suggest that neutrophil elastase inhibition could protect blood-brain barrier function in acute cerebral ischemia by augmentation of angiopoietin-1 expression and survival of endothelial cells.

Hypertension Research (2010) 33, 703-707; doi:10.1038/hr.2010.58; published online 20 May 2010

Keywords: acute cerebral ischemia; angiopoietin-1; brain edema endothelial cells; neutrophil elastase inhibitor

\section{INTRODUCTION}

Neutrophils have a major role in acute inflammatory reactions. In the early phase of cerebral ischemia, neutrophils accumulate in the damaged lesion of the ischemic brain tissue. ${ }^{1,2}$ These activated neutrophils can cause critical neuropathological changes in the brain tissue via several mechanisms, including the release of neutrophil elastase (NE) ${ }^{3,4}$ Therefore, inhibition of NE released from neutrophils could be a useful strategy for the treatment of acute stroke. In fact, several investigators have reported that serum NE levels are elevated in patients with acute ischemic stroke. ${ }^{5,6}$ Sivelestat is one of the agents which can specifically inhibit $\mathrm{NE}^{7}$ and it is used for the treatment of acute lung injury patients or acute respiratory distress syndrome patients. ${ }^{8,9}$ Although a few reports showed the possibility that sivelestat might reduce cerebral ischemic damage, ${ }^{10,11}$ the detailed protective mechanism of sivelestat in brain tissue has not yet been elucidated.

In this study, we have investigated the effects of sivelestat on acute cerebral ischemia. Especially, we focused on its protective mechanism for blood-brain barrier (BBB) and neurovascular endothelial cells (EC), because BBB disruption and brain edema can cause a critical event in the acute phase of ischemic stroke such as hemorrhagic alternation or secondary tissue damage in other normal regions due to mechanical stress, and subsequently worsen the prognosis.

\section{METHODS}

Animals

The study was approved by the Animal Care Committee of Gifu University Graduate School of Medicine. Age-matched 8-week-old male C57BL/6 mice weighing 24-27 g (SLC, Shizuoka, Japan) were used and housed under diurnal lighting conditions.

Focal cerebral ischemia model

Mice were anesthetized with $2.0 \%$ isoflurane (Nissan Kagaku, Tokyo, Japan), followed by $1 \%$ isoflurane in $70 \% \mathrm{~N}_{2} \mathrm{O}$ and $30 \% \mathrm{O}_{2}$ using an animal general anesthesia machine (Soft Lander; Sin-ei Industry, Saitama, Japan), and placed in the supine position with spontaneous respiration. Rectal temperature was maintained at $37.0-37.5^{\circ} \mathrm{C}$ by a heating pad and a heating lamp. Left middle cerebral artery occlusion (MCAO) with a filament was carried out as previously described. ${ }^{12}$

Drug administration

Sivelestat (ONO-5046; Ono Pharmaceutical, Osaka, Japan) was dissolved in PBS and administered twice (intraperitoneally just after MCAO, and

\footnotetext{
${ }^{1}$ Department of Neurosurgery, Gifu University Graduate School of Medicine, Gifu, Japan and 2Department of Cell Signaling, Gifu University Graduate School of Medicine, Gifu, Japan

${ }^{3}$ These authors contributed equally to this work.

Correspondence: Dr Y Ikegame, Department of Neurosurgery, Gifu University Graduate School of Medicine, 1-1 Yangido, Gifu 501-1194, Japan.

E-mail: ikegame-nsu@umin.ac.jp or Dr S-i Hayashi, Department of Cell Signaling, Gifu University Graduate School of Medicine, Gifu 501-1194, Japan.

E-mail: Shin559182@aol.com
}

Received 9 January 2010; revised 19 February 2010; accepted 22 February 2010; published online 20 May 2010 
subcutaneously $12 \mathrm{~h}$ after occlusion) to groups of mice at doses of 0 (vehicle) and $50 \mathrm{mg} \mathrm{kg}^{-1}$. Mice in the sham group only underwent ligation of the left common cervical artery.

\section{Assessment of neurological deficit}

The neurological deficit just after and $24 \mathrm{~h}$ after MCAO was scored using the following scale as previously reported: ${ }^{12} 0$, no observable neurological deficit (normal); 1, failure to extend the right forepaw (mild); 2, circling to the contralateral side (moderate); 3 , loss of walking or righting reflex (severe). All measurements were performed by researchers blind to the treatment of each animal.

\section{Evaluation of infarct area and edema volume}

To analyze the infarct area and volume at $24 \mathrm{~h}$ after MCAO, ${ }^{12}$ mice were killed by intraperitoneal injection of sodium pentobarbital (Dainippon Sumitomo Pharma, Osaka, Japan). The removed forebrain was sectioned coronally at 2-mm intervals. The sections were stained with 2\% 2,3,5-triphenyltetrazolium chloride (TTC, Sigma, Diagnostics, St Louis, MO, USA), and recorded as digital images. The areas of infarct (TTC-unstained area) and ipsilateral/contralateral hemispheres were quantified using imaging software, ImageJ (National Institutes of Health, USA). The infarct volume and brain swelling were calculated using formulas specified in a previous report. ${ }^{13}$

\section{Quantitative measurements of Evans Blue}

A quantitative method was used to measure the amount of Evans Blue dye extravasation as previously reported with some modifications. ${ }^{14}$ Following $24 \mathrm{~h}$ of MCAO, Evans Blue dye ( $0.1 \mathrm{ml}$ of $2 \%$ solution in saline) was injected into mice via a tail vein. After transcardiac brain perfusion with saline, the divided brains were cut into the left and right hemispheres. Brain tissue was weighed, the extracted dye was measured with a luminescence spectrometer (ImmunoMini NJ-2300, InterMed, Tokyo, Japan), and the tissue content of Evans Blue was quantified from a standard curve derived from known amounts of dye.

\section{In vivo lectin staining of brain microvessels}

EC were prelabeled by injecting $100 \mu \mathrm{g}$ fluorescein-labeled Griffonia simplicifolia lectin I-isolectin B4 (GSL-IB4, Vector Laboratories, Burlingame, CA, USA) into a tail vein at $24 \mathrm{~h}$ after MCAO. The GSL-IB4 was diluted in $0.1 \mathrm{ml}$ saline. Mice were perfused transcardially with $4 \%$ paraformaldehyde. Brains were cut into $10-\mu \mathrm{m}$ slices, and examined under a fluorescence microscope (Axiovert 200, Carl Zeiss, Thornwood, NY, USA).

\section{Immunohistochemical staining}

Mice were perfused transcardially with $4 \%$ paraformaldehyde at $24 \mathrm{~h}$ after MCAO. The brain was removed, fixed in $4 \%$ paraformaldehyde for $5 \mathrm{~h}$, frozen, and sectioned coronally at a thickness of $10 \mu \mathrm{m}$ on a cryostat. The primary antibody against ANGPT1 (1:200, Santa Cruz Biotechnology, Santa Cruz, CA, USA) and a Vecterstain Elite ABC kit (Vector Laboratories) were used, followed by counterstaining with hematoxylin. The stained sections were examined under an Axiovert 200 microscope. These sections were also photographed as gray scale images, and the immunoreactivity of ANGPT1 was calculated using ImageJ software.

\section{Cell survival assay}

Human brain microvascular endothelial cells (HBMVEC) were obtained from Cell Systems (Kirkland, WA, USA). They were plated at a density of $1 \times 10^{5}$ cells per well on 6-well culture plates and incubated in EBM-2 medium with endothelial growth supplements (Lonza, Walkersville, MD, USA) for $6 \mathrm{~h}$ (at $37^{\circ} \mathrm{C}$, in a humidified atmosphere containing $5 \% \mathrm{CO}_{2}$ ). Elastase (Calbiochem, EMD Chemicals, La Jolla, CA, USA) and sivelestat were added into the medium and EC were cultured for $24 \mathrm{~h}$. After removal of the conditioned medium, $0.4 \%$ trypan blue exclusion analysis was performed.

\section{Statistical analysis}

Statistical comparisons were made by Student's $t$-test or Mann-Whitney $U$-test (for comparison of neurological deficit scores). $P$-values of $<0.05$ were considered to indicate statistical significance. Data are presented as mean \pm s.e.m.

\section{RESULTS}

Sivelestat a NE inhibitor protects against acute ischemic brain damage

Acute cerebral ischemia model was performed by the occlusion of mouse left middle cerebral artery by a filament as previously described. ${ }^{12}$ We designed an experimental protocol that requires twice injection of sivelestat within $24 \mathrm{~h}$ after MCAO to make it come near continuous infusion (Figure 1). Administration of sivelestat significantly attenuated the neurological deficit score in the acute phase of MCAO compared with that in the vehicle group (Figure 2). Analysis of coronal brain sections at $24 \mathrm{~h}$ after MCAO revealed that sivelestat reduced both infarct volume (Figure 3a) and ipsilateral edema volume (Figure $3 b$ ).

We next examined the effect of sivelestat on vascular permeability in murine brain, because the reduction rate of swelling $(67.8 \%)$ was much higher than that of infarct volume $(43.7 \%)$ after sivelestat treatment. MCAO treatment caused the gain of the injured hemisphere weight (Figure 4a), due to edema. Sivelestat effectively prevented the increase of hemisphere weight. Furthermore, the content of leaked Evans Blue after $24 \mathrm{~h}$ of MCAO in the left and right hemispheres was quantified and standardized by the weight of each hemisphere. As shown in Figure 4b, MCAO increased Evans Blue content in the ipsilateral hemisphere as compared with that in the contralateral hemisphere. The NE inhibitor significantly reduced the amount of Evans Blue content in the injured hemisphere. These data indicate the possibility that NE inhibitors may prevent $\mathrm{BBB}$ disruption and subsequent leakage of water and serum. There were no significant differences in physiological parameters (mean arterial blood pressures,

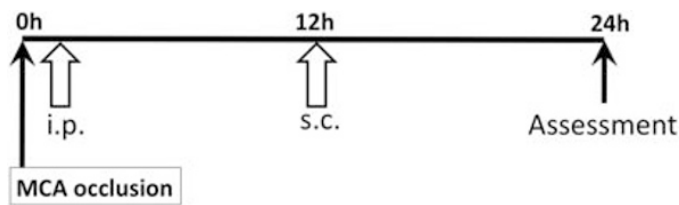

Figure 1 Protocol for drug administration. Intraperitoneal (i.p) and subcutaneous (s.c.) injections of vehicle or sivelestat $\left(50 \mathrm{mg} \mathrm{kg}^{-1}\right)$ were performed at just after MCAO and $12 \mathrm{~h}$ after occlusion, respectively. All assessments or experiments carried out after $24 \mathrm{~h}$ of MCAO.

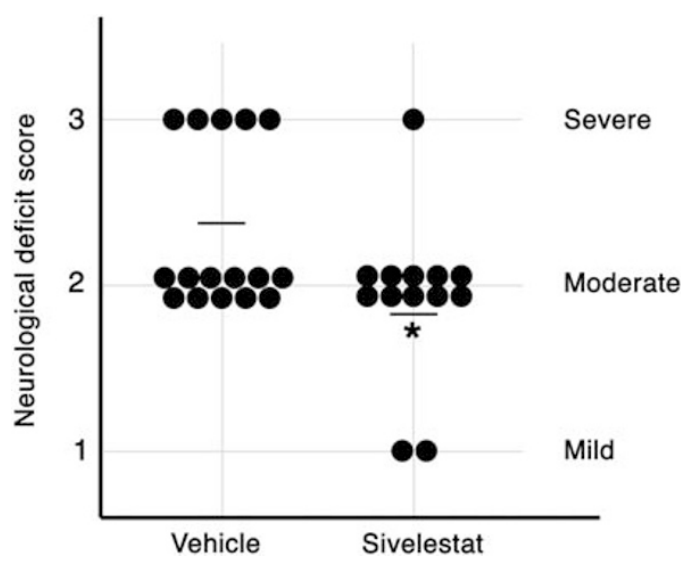

Figure 2 Neurological deficit scores in mice with MCAO and sivelestat treatment. Mice with score 2 just after MCAO were selected for assessment. Dots in graph represent deficit score of each mouse after $24 \mathrm{~h}$ of MCAO. Horizontal bars indicate average deficit score in sivelestat-treated group and vehicle group. ${ }^{*} P<0.05$ between vehicle group $(n=16)$ and sivelestat group $(n=13)$. 

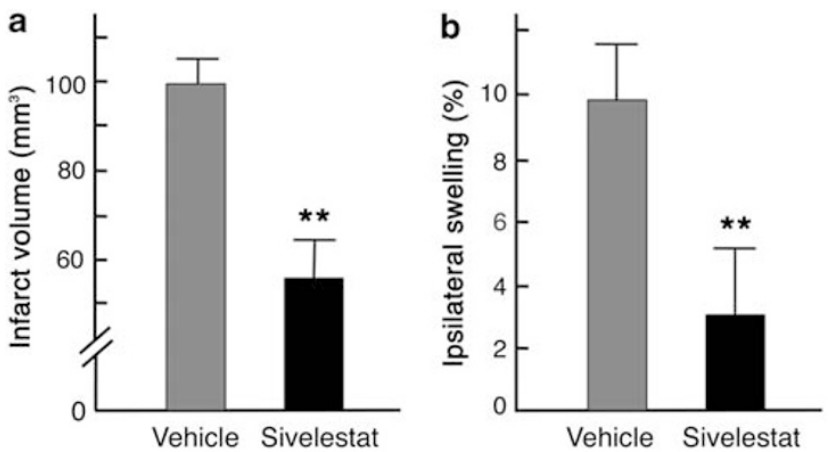

Figure 3 Sivelestat reduces infarct volume and swelling after MCAO. Infarct volume (a) and swelling volume (b) were quantified in each mouse after $24 \mathrm{~h}$ of MCAO. Values are expressed as means \pm s.e.m. Swelling volume in the left hemisphere was compared with that in the right hemisphere. ${ }^{* *} P<0.01$ between vehicle group $(n=16)$ and sivelestat group $(n=13)$.
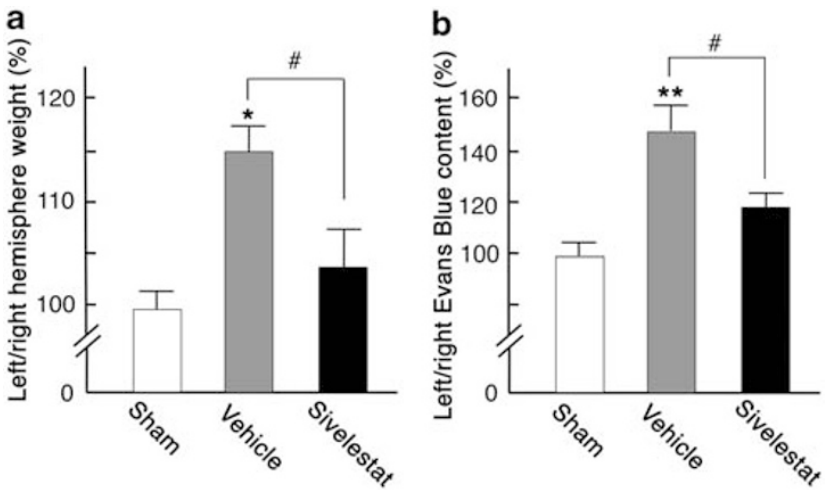

Figure 4 Sivelestat reduces vasogenic edema after MCAO. Percent left/right hemisphere weight (a) and Evans Blue content (b) were compared among the sham group $(n=3)$, vehicle group $(n=14)$, and sivelestat group $(n=11)$ after $24 \mathrm{~h}$ of MCAO. Note that $100 \%$ mean values of left hemisphere are equal to those of right hemisphere. Values are expressed as mean \pm s.e.m. ${ }^{*} P<0.05$ or ${ }^{* *} P<0.01$ vs sham-operated group. ${ }^{\#} P<0.05$ vs. vehicle group.

pulse rate, $\mathrm{pH}$, partial pressures of $\mathrm{CO}_{2}$ in arterial blood, partial pressures of $\mathrm{O}_{2}$ in arterial blood) among the experimental groups (data not shown).

\section{ANGPT1 expression and morphology of microvessels in mouse brain after MCAO}

We next investigated ANGPT1 expression in mouse brain tissue by immunohistochemical staining, because ANGPT1 might be an effective factor for BBB protection after cerebral infarction. ${ }^{14}$ Interestingly, ANGPT1 expression was increased in the ischemic margin area in sivelestat-treated mice compared with that in the vehicle group and to that in the intact area of untreated mice, whereas no change in its expression was observed in the ischemic margin area in the vehicle group compared with that in the intact area of untreated mice (Figure 5a, upper panels; Figure 5b). To further investigate whether sivelestat affects the morphology of microvessels in ischemic brain tissue, fluorescein-labeled GSL-IB4 (isolectin-B4) was intravenously injected before killing, and brain tissue sections were examined with a fluorescence microscope. In the vehicle group, most of the microvessels in the infarcted area were structurally destroyed (Figure 5a, middle panels). On the other hand, microvessels in the boundary zone were well maintained in the sivelestat-treated group (Figure $5 \mathrm{a}$, right panels).
Inhibition of NE-induced human EC damage by sivelestat

To confirm the mechanisms of $\mathrm{BBB}$ protection by sivelestat in vitro, the direct effect of NE inhibition on human brain EC was examined. HBMVEC were cultured with various concentrations of $\mathrm{NE}$ in the absence or presence of sivelestat for $24 \mathrm{~h}$. As shown in Figure 6, NE concentration dependently decreased the number of EC. The NE concentrations higher than $250 \mathrm{ng} \mathrm{m}^{-1}$, conditions corresponding to the blood level of patients with ischemic stroke, ${ }^{6}$ significantly decreased the cell number of EC. In contrast, clinical doses of sivelestat concentration dependently reversed EC from NE-induced reduction of the cell number. These results indicate that sivelestat protects HBMVEC from NE-induced damage.

\section{DISCUSSION}

In this study, sivelestat successfully reduced brain infarction area, edema volume and BBB hyperpermeability in the acute phase of murine MCAO. It further preserved neurological function in the damaged brain. Interestingly, the sivelestat-treated group showed higher ANGPT1 expression and better maintained structure of microvessels in the ischemic margin area as compared with the vehicle group.

Several investigators have shown that neutrophils accumulate in the ischemic area within $24 \mathrm{~h}$ after the onset of brain infarction in both experimental models and clinical studies. ${ }^{1,2,15}$ In this situation, activated neutrophils can cause neurovascular injury by releasing free radicals, inflammatory cytokines or destructive enzymes. ${ }^{3,4,16}$ Among them, NE, one of the proteases from neutrophils, induces not only systemic harmful reaction via humoral mechanism ${ }^{4}$ but also local damages such as neuronal cell death ${ }^{17-19}$ and increase in BBB permeability. ${ }^{20,21}$ Therefore, NE inhibition can be beneficial to attenuate these inflammatory damages. On the rodent brain tissue after cerebral ischemia, immunohistochemical staining have revealed that NE distributes in both perivascular space and the extracellular space in the neuronal layer. ${ }^{11,22}$ In the clinical setting, brain infarction patients with hemorrhagic alteration showed higher blood NE levels (730$1320 \mathrm{ng} \mathrm{ml}^{-1}$ ), vs. less than $250 \mathrm{ng} \mathrm{ml}^{-1}$ in patients without hemorrhagic alteration ${ }^{6}$ and around $30-70 \mathrm{ng} \mathrm{ml}^{-1}$ in patients without ischemic stroke, ${ }^{5,23}$ suggesting that the level of $\mathrm{NE}$ is likely to be related to prognosis. In addition, hemorrhagic alternation is related to $\mathrm{BBB}$ disruption. Then, we hypothesized that a specific NE inhibitor, sivelestat, ${ }^{7}$ may be effective for BBB protection, which is necessary for the prevention of critical secondary injury after cerebral ischemia such as brain herniation.

Our in vivo data showed that administration of sivelestat not only preserved neurological function after acute cerebral ischemia but also reduced brain infarction size. In support of this, a report showed that sivelestat attenuated photochemical reaction-induced brain infarction. ${ }^{10}$ Several mechanisms can be considered with respect to causes for reduction of infarct volume: neuroprotective effect and/or BBB protective effect. As for direct protective effects on neural cells, some investigators indicated that sivelestat could prevent apoptosis of neuronal cells induced by ischemia. ${ }^{11,19,24}$ On the other hand, a mechanism via $\mathrm{BBB}$ protection is indirect: preserved microcirculation supply brain tissue with blood enough to survive. Our data also suggest that sivelestat attenuates $\mathrm{BBB}$ disruption by reducing $\mathrm{BBB}$ hyperpermeability (Figure $4 \mathrm{~b}$ ). Similarly, another examination using a different NE inhibitor succeeded in preventing BBB breakdown and showed decrease in infarction volume. ${ }^{22}$ These findings show that NE inhibition is useful for BBB protection.

In seeking to define the mechanism underlying $\mathrm{BBB}$ protection by sivelestat, we also examined ANGPT1 expression in the ischemic brain 
a
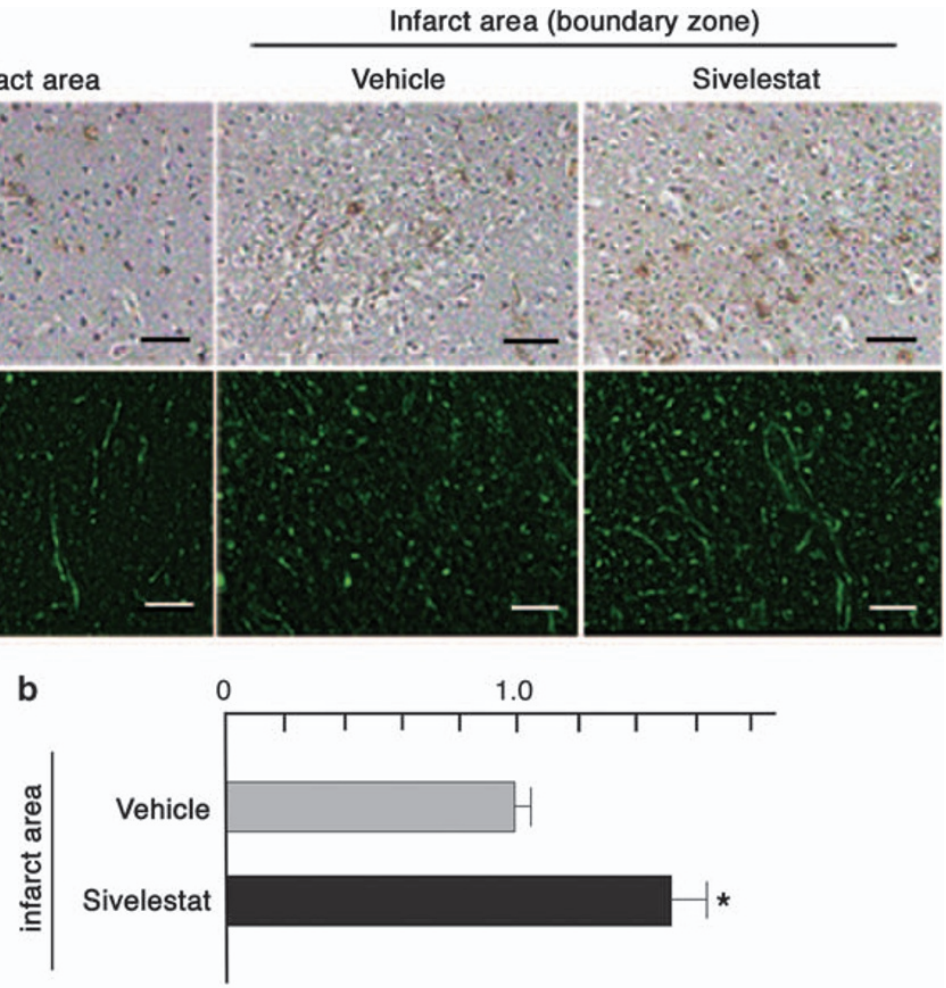

Relative density of ANGPT1 expression

Figure 5 Histological changes in neuromicrovessels after MCAO and sivelestat treatment. (a) Representative immunohistochemical images of ANGPT1 expression around microvessels in intact area (left panels) and ischemic boundary zone (middle and right panels) in cortex (upper panels). Histological changes in neuromicrovessels after MCAO and sivelestat treatment. Representative fluorescent images of isolectin-B4-positive vessels in intact area (left panels) and ischemic boundary zone (middle and right panels) in cortex (lower panels). Scale bar, $50 \mu \mathrm{m}$. (b) Immunoreactivates of ANGPT1 in the sivelestat-treated group relative to those in the vehicle group. ${ }^{*} P<0.05$ vs. vehicle.

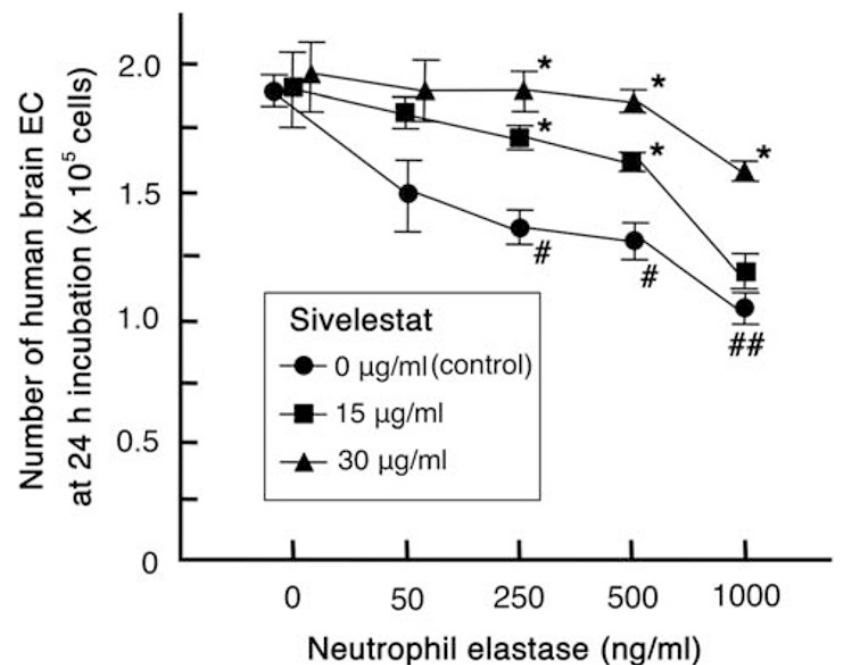

Figure 6 In vitro effects of sivelestat on human brain EC. The cell number of HBMVEC were counted after $24 \mathrm{~h}$ of incubation with sivelestat $(\bullet$; $\left.0 \mu \mathrm{g} \mathrm{m}^{-1}, \mathbf{\square} ; 15 \mu \mathrm{g} \mathrm{ml}^{-1}, \mathbf{\Delta} ; 30 \mu \mathrm{g} \mathrm{ml}^{-1}\right)$ and $\mathrm{NE}(0,50,250,500$ or $1000 \mathrm{ng} \mathrm{ml}^{-1}$ ). Each dot and error bar express mean \pm s.e.m. $n=4$ per group. Representative data from at least three separate experiments are shown. ${ }^{*} P<0.05$ vs. control. ${ }^{\#} P<0.05$ and ${ }^{\# \#} P<0.01$ vs. 0 ng ml ${ }^{-1} \mathrm{NE}$.

for the following reasons: (i): ANGPT1, a crucial factor for vascular development, weakened peripheral vascular leakage caused by inflammatory agents ${ }^{25,26}$ and target cell junctions. ${ }^{27}$ (ii): ANGPT1 expression in the brain was downregulated in acute ischemia. ${ }^{28}$ (iii): ANGPT1 prevented BBB leakage after focal cerebral ischemia. ${ }^{14}$ Strikingly, our data showed that sivelestat enhanced ANGPT1 expression in the ischemic brain. Although it is not clarified which are the cell types that mainly secrete ANGPT1, astrocytes or pericytes are thought as major candidates. ${ }^{29,30}$ According to previous reports, NE widely invades into ischemic area beyond perivascular space. ${ }^{11,22}$ The observation implies that the spread NE can injure the source cells of ANGPT1 such as astrocytes and/or pericytes. It is possible that injury of the source cells consequently cause downregulation of ANGPT1 in the ischemic zone. Moreover, it is known that sivelestat suppresses NE expression in brain tissue after ischemia. ${ }^{11}$ Taken together, upregulation of ANGPT1 by sivelestat is presumably due to rescuing various source cells in brain tissue from non-specific proteolytic insult of NE. Subsequently, BBB protection via ANGPT1-related mechanisms also could lead to maintain the architecture of microvessels (Figure 5a). Collectively, our histological findings indicate that preserved ANGPT1 expression by means of sivelestat is effective to maintain both the function and structure of BBB.

In our in vitro study using HBMVEC, NE significantly worsened EC survival when the concentration was more than $250 \mathrm{ng} \mathrm{ml}^{-1}$, corresponding to those found in the blood of patients with cerebral infarction at acute phase. However, sivelestat $\left(15 \mu \mathrm{g} \mathrm{ml}^{-1}, 30 \mu \mathrm{g} \mathrm{ml}^{-1}\right)$ effectively rescued HBMVEC from NE-induced damage, indicating that clinical doses of sivelestat can prevent severe BBB disruption enough to cause hemorrhagic alteration. According to Phase I and Phase II clinical trials in Japan, a steady state blood concentration of sivelestat showed around $10-30 \mu \mathrm{g} \mathrm{ml}^{-1}$. Although the dose did not 
show any adverse effects in previous clinical investigations, our results showed that administration of sivelestat at the clinical dose might have therapeutic usefulness for BBB protection after acute cerebral infarction.

In conclusion, NE inhibition by sivelestat could attenuate $\mathrm{BBB}$ disruption in acute brain ischemia through the enhancement of ANGPT1 production and EC survival, which might decrease brain tissue damage and neurological deficit in patients with acute brain ischemia.

\section{CONFLICT OF INTEREST}

The authors have no conflict of interest.

\section{ACKNOWLEDGEMENTS}

This work was supported in part by a grant from the NaSNeLC research committee (Y Ikegame) and by a dean's grant from Gifu University

(S-i Hayashi)

1 Kato H, Kogure K, Liu XH, Araki T, Itoyama Y. Progressive expression of immunomolecules on activated microglia and invading leukocytes following focal cerebral ischemia in the rat. Brain Res 1996; 734: 203-212.

2 Kochanek PM, Hallenbeck JM. Polymorphonuclear leukocytes and monocytes/ macrophages in the pathogenesis of cerebral ischemia and stroke. Stroke 1992; 23 1367-1379

3 Fagan SC, Hess DC, Hohnadel EJ, Pollock DM, Ergul A. Targets for vascular protection after acute ischemic stroke. Stroke 2004; 35: 2220-2225.

4 Frangogiannis NG. Chemokines in ischemia and reperfusion. Thromb Haemost 2007; 97: 738-747.

5 Cojocaru IM, Cojocaru M, Burcin C. Evaluation of granulocyte elastase as a sensitive diagnostic parameter of inflammation in first ischemic stroke. Rom J Intern Med 2006; 44: 317-321.

6 Iwatsuki K, Kumura E, Yoshimine T, Yamamoto K, Sato M, Hayakawa T. Increase in jugular levels of polymorphonuclear neutrophil elastase in patients with acute cerebral infarction. Neurol Res 1998; 20: 397-402.

7 Kawabata K, Suzuki M, Sugitani M, Imaki K, Toda M, Miyamoto T. ONO-5046, a novel inhibitor of human neutrophil elastase. Biochem Biophys Res Commun 1991; 177 814-820.

8 Zeiher BG, Matsuoka S, Kawabata K, Repine JE. Neutrophil elastase and acute lung injury: prospects for sivelestat and other neutrophil elastase inhibitors as therapeutics. Crit Care Med 2002; 30: S281-S287.

9 Hayakawa M, Katabami K, Wada T, Sugano M, Hoshino H, Sawamura A, Gando S. Sivelestat (selective neutrophil elastase inhibitor) improves the mortality rate of sepsis associated with both acute respiratory distress syndrome and disseminated intravascular coagulation patients. Shock 2010; 33: 14-18.

10 Shimakura A, Kamanaka Y, Ikeda Y, Kondo K, Suzuki Y, Umemura K. Neutrophil elastase inhibition reduces cerebral ischemic damage in the middle cerebral artery occlusion. Brain Res 2000; 858: 55-60.

11 Matayoshi H, Hirata T, Yamashita S, Ishida K, Mizukami Y, Gondo T, Matsumoto M, Sakabe T. Neutrophil elastase inhibitor attenuates hippocampal neuronal damage after transient forebrain ischemia in rats. Brain Res 2009; 1259: 98-106.

12 Yamashita K, Kotani Y, Nakajima Y, Shimazawa M, Yoshimura S, Nakashima S, Iwama T, Hara H. Fasudil, a Rho kinase (ROCK) inhibitor, protects against ischemic neuronal damage in vitro and in vivo by acting directly on neurons. Brain Res 2007; 1154: 215-224.

13 Hara H, Friedlander RM, Gagliardini V, Ayata C, Fink K, Huang Z, Shimizu-Sasamata M, Yuan J, Moskowitz MA. Inhibition of interleukin $1 \beta$ converting enzyme family proteases reduces ischemic and excitotoxic neuronal damage. Proc NatI Acad Sci USA 1997; 94: 2007-2012.

14 Zhang ZG, Zhang L, Croll SD, Chopp M. Angiopoietin-1 reduces cerebral blood vessel leakage and ischemic lesion volume after focal cerebral embolic ischemia in mice. Neuroscience 2002; 113: 683-687.

15 Price CJ, Menon DK, Peters AM, Ballinger JR, Barber RW, Balan KK, Lynch A, Xuereb JH, Fryer T, Guadagno JV, Warburton EA. Cerebral neutrophil recruitment, histology, and outcome in acute ischemic stroke: an imaging-based study. Stroke 2004; 35: 1659-1664.

16 Kochanek PM, Hallenbeck JM. Polymorphonuclear leukocytes and monocytes/ macrophages in the pathogenesis of cerebral ischemia and stroke. Stroke 1992; 23: 1367-1379.

17 Dinkel K, Dhabhar FS, Sapolsky RM. Neurotoxic effects of polymorphonuclear granulocytes on hippocampal primary cultures. Proc Natl Acad Sci USA 2004; 101: 331-336.

18 Tonai T, Shiba K, Taketani Y, Ohmoto Y, Murata K, Muraguchi M, Ohsaki H, Takeda E, Nishisho T. A neutrophil elastase inhibitor $(0 N 0-5046)$ reduces neurologic damage after spinal cord injury in rats. J Neurochem 2001; 78: 1064-1072.

19 Yamauchi T, Sawa Y, Sakurai M, Hiroshi T, Matsumiya G, Abe K, Matsuda H. ONO-5046 attenuation of delayed motor neuron death and effect on the induction of brain-derived neurotrophic factor, phosphorylated extracellular signal-regulated kinase, and caspase 3 after spinal cord ischemia in rabbits. J Thorac Cardiovasc Surg 2006; 131: 644-650.

20 Armao D, Kornfeld M, Estrada EY, Grossetete M, Rosenberg GA. Neutral proteases and disruption of the blood-brain barrier in rat. Brain Res 1997; 767: 259-264.

21 Nagy Z, Kolev K, Csonka E, Vastag M, Machovich R. Perturbation of the integrity of the blood-brain barrier by fibrinolytic enzymes. Blood Coagul Fibrinolysis 1998; 9: 471-478.

22 Stowe AM, Adair-Kirk TL, Gonzales ER, Perez RS, Shah AR, Park TS, Gidday JM. Neutrophil elastase and neurovascular injury following focal stroke and reperfusion. Neurobiol Dis 2009; 35: 82-90.

23 Amaro A, Gude F, Gonzalez-Juanatey R, Iglesias C, Fernandez-Vazquez F, Garcia-Acuna $\mathrm{J}$, Gil M. Plasma leukocyte elastase concentration in angiographically diagnosed coronary artery disease. Eur Heart J 1995; 16: 615-622.

24 Iwamoto S, Higashi A, Ueno T, Goto M, Iguro Y, Sakata R. Protective effect of sivelestat sodium hydrate (ONO-5046) on ischemic spinal cord injury. Interact Cardiovasc Thorac Surg 2009; 8: 606-609.

25 Thurston G, Suri C, Smith K, McClain J, Sato TN, McDonald DM. Leakage-resistant blood vessels in mice transgenically overexpressing angiopoietin-1. Science 1999; 286: 2511-2514.

26 Thurston G, Rudge JS, Ioffe E, Zhou H, Ross L, Croll SD, Glazer N, Holash J, McDonald DM, Yancopoulos GD. Angiopoietin-1 protects the adult vasculature against plasma leakage. Nat Med 2000; 6: 460-463.

27 Gamble JR, Drew J, Trezise L, Underwood A, Parsons M, Kasminkas L, Rudge J, Yancopoulos G, Vadas MA. Angiopoietin-1 is an antipermeability and anti-inflammatory agent in vitro and targets cell junctions. Circ Res 2000; 87: 603-607.

28 Zhang ZG, Zhang L, Tsang W, Soltanian-Zadeh H, Morris D, Zhang R, Goussev A, Powers $C$, Yeich T, Chopp M. Correlation of VEGF and angiopoietin expression with disruption of blood-brain barrier and angiogenesis after focal cerebral ischemia. J Cereb Blood Flow Metab 2002; 22: 379-392.

29 Lee SW, Kim WJ, Choi YK, Song HS, Son MJ, Gelman IH, Kim YJ, Kim KW. SSeCKS regulates angiogenesis and tight junction formation in blood-brain barrier. Nat Med 2003; 9: 900-906.

30 Hori S, Ohtsuki S, Hosoya K, Nakashima E, Terasaki T. A pericyte-derived angiopoietin-1 multimeric complex induces occludin gene expression in brain capillary endothelial cells through Tie-2 activation in vitro. J Neurochem 2004; 89: 503-513. 